\title{
A Random Differential Equation based method for a $d$-dimensional PDE of convection-diffusion type
}

\author{
Hugo de la Cruz ${ }^{1}$ \\ Escola de Matemática Aplicada, Fundação Getulio Vargas, Rio de Janeiro, Brazil \\ Christian Olivera ${ }^{2}$ \\ Departamento de Matemática, Universidade Estadual de Campinas, Brazil
}

\begin{abstract}
We consider $d$-dimensional PDEs of convention-diffusion type with at most Hölder continuous coefficients. We construct an stochastic numerical method for the Monte Carlo integration of this kind of equations. The proposed approach is based on the probabilistic representation of this deterministic PDE through the solution of an associated stochastic transport equation, which remarkably can be efficiently integrated without considering the standard assumptions that typically are needed by convectional numerical integrators for solving the underlying PDE. Results on the convergence of the proposed method and details on its implementation are presented.
\end{abstract}

Keywords. Convection-Diffusion PDEs, Numerical Approximation, Probabilistic Numerical Methods, Monte Carlo Methods, Random Differential Equations, Random Euler Method

\section{Introduction}

Many initial value problems for Partial Differential Equations (PDEs) that arise in applications usually contain rough, non-smooth coefficients defining the PDE. Consequently the application of conventional numerical integrators for such equations does not make any sense [8]. This is the case of the convection-diffusion equation in $\mathbb{R}^{d}$

$$
\begin{aligned}
u_{t}(t, x)+b(t, x) \cdot \nabla u(t, x)-\frac{1}{2} \Delta u(t, x) & =0 \\
u(0, x) & =f(x),
\end{aligned}
$$

where $b:\left[\begin{array}{ll}0 & T\end{array}\right] \times \mathbb{R}^{d} \rightarrow \mathbb{R}^{d}$ is measurable, bounded and $\alpha$-Hölder continuous in space uniformly in time, for some $\alpha \in(0,1)$.

Since convection-diffusion is an essential constituting part of useful practical models, it has been extensively studied and much research has been carried out concerning the numerical approximation of equation (1). In fact, it is well known that there are different ways to discretize convection-diffusion equations e.g., by using finite element and finite

\footnotetext{
${ }^{1}$ hugo.delacruz@fgv.br

${ }^{2}$ colivera@ime.unicamp.br
} 
difference methods including fully discrete schemes, Methods of lines, Rothe's method, Exponential Fitting, Meshless methods, IMEX methods, etc (see e.g., [10], [3]). However, when the coefficient $b$ in (1) is rough (for instance not differentiable or even continuous) standard numerical integrators fails to work properly due to the assumptions requisite for convergence are not satisfied [8]. That is why is necessary to resort to alternative methods and mathematical tools for devising new integrators specially tailored for equation (1) when there is a lack of regularity in the coefficient $b$.

The objective of this work is to construct a numerical integrator for the approximation of the PDE (1) when $b$ is not sufficiently smooth, in particular when $b$ is only $\alpha$-Hölder continuous in space uniformly in time, for some $\alpha \in(0,1)$. The approach we follows is based on the probabilistic representation of this PDE through the solution of an associated stochastic transport equation, which can be efficiently integrated via the solution of a suitable Random Differential Equations (RDE) without considering the standard assumptions that typically are needed by conventional numerical integrators for solving the underlying PDE.

The emergence of modern computing machines with ever higher speed and capacity have contributed to a growing interest in stochastic approaches for deterministic PDEs. It has played an important role in situations where using deterministic integrators become ineffective due to huge volume of computations (for example, for $d>3$ ) and when it is desired to compute the values of the solution in only few points. In particular for equation (1), the solution is given by [4]

$$
u(t, x)=\mathbb{E}\left(f(W(t)) \exp \left(-\int_{0}^{t} b(W(s)) d W(s)-\frac{1}{2} \int_{0}^{t}|W(s)|^{2} d s\right)\right),
$$

where $\mathbb{E}(X)$ stand for the expected value of the random variable $X$ and $W(s)$ is a Wiener processes (often called Brownian motion). That is $\{W(t) ; t \geq 0\}$ is a random processes with continuous path, defined by the properties: $W(s)$ is Gaussian distributed (i.e., $W(s) \sim N(0, t))$, increments $W(t)-W(s) \sim N(0, t-s)$, with $0 \leq s \leq t$, and are independent on non overlapping subintervals (i.e., for any $0=t_{0}<t_{1}<\cdots<t_{N}$, $\left\{W\left(t_{i}\right)-W\left(t_{i-1}\right) ; 1 \leq i \leq N\right\}$ are independent random variables) and $W(0)=0$ with probability 1 . The Itô stochastic integral is the mean-square $(m . s)$ limit [1]

$$
\int_{0}^{t} b(W(s)) d W(s):=(m . s) \lim _{N \rightarrow \infty} \sum_{k=0}^{N-1} b\left(W\left(t_{k}\right)\right)\left(W\left(t_{k+1}\right)-W\left(t_{k}\right)\right),
$$

taken over partitions $0=t_{0}<t_{1}<\cdots<t_{N}=t$ of the interval $[0 t]$, with $\max _{k=0, \ldots, N-1}\left(t_{k+1}-\right.$ $\left.t_{k}\right) \rightarrow 0$ as $N \rightarrow \infty$.

However, the proof of this representation is based on the Ito-Formula from stochastic calculus -or alternatively in the classical Feynman-Kac Formula- (see e.g., [4]) and consequently is valid under a set of smoothness hypothesis on the function $b$, that are not fulfilled by (1). Even if it is proven in some other less restricted way that this representation is still valid to determinate $u(t, x)$, the integral $\int_{0}^{t}|W(s)|^{2} d s$ needs to be computed by 
quadrature, for which is implicitly assumed that the function to be integrated (in this case $\left.|W(s)|^{2}\right)$ is smooth enough. This is a serious problem since the Wiener processes $W(s)$ is nowhere-differentiable almost surely [1]. The novelty of the present paper precisely consist in devising a numerical integrator for (1) without very restrictive smoothness hypothesis. Basically only assuming $\alpha$-Hölder continuity of $b$.

The paper is organized as follows. In the next section a probabilistic representation for the solution of the PDE is presented and a associated RDE is considered. Section 3 presents the deduction and convergence of the proposed method. Also, a detailed algorithm concerning the implementation of the proposed method is described.

\section{A Probabilistic Representation for the Equation (1)}

\subsection{Representation via an Stochastic Transport Equation}

Let $\left(\Omega, \mathcal{F},\left(\mathcal{F}_{t}\right), \mathbb{P}\right)$ be a filtered probability space. Consider the stochastic transport equation $[2]$

$$
v(t, x)=f(x)-\int_{0}^{t} b(s, x) \cdot \nabla v(s, x) d s-\sum_{j=0}^{d} \int_{0}^{t} \nabla v(s, x) \circ d W^{j}(s),
$$

where $W(t)=\left(W^{1}(t),, \ldots, W^{d}(t)\right)$ is a standard $\mathcal{F}_{t}$-adapted $d$-dimensional Wiener processes on $\left(\Omega, \mathcal{F},\left(\mathcal{F}_{t}\right), \mathbb{P}\right)$ and the stochastic differentials $\circ d W^{j}(t)$ are understood in the Stratonovich sense [1].

Under the assumptions for $b$ in the previous section, F. Flandoli et al., [5] proved that the solution of $(1)$ in $(t, x)$ satisfies

$$
u(t, x)=\mathbb{E}(v(t, x)) .
$$

On the other hand, the solution of $(2)$ in $(t, x)$ satisfies

$$
v(t, x)=f\left(\phi_{0, t}^{-1}(x)\right), \quad t \geq 0
$$

where the value $\phi_{0, t}^{-1}(x) \in \mathbb{R}^{d}$ is such that the solution of

$$
\begin{aligned}
d X(t) & =b(t, X(t)) d t+d W(t) \\
X(0) & =\phi_{0, t}^{-1}(x),
\end{aligned}
$$

satisfies $X(t)=x$.

Thus,

$$
u(t, x)=\mathbb{E}(v(t, x))=\mathbb{E}\left(f\left(\phi_{0, t}^{-1}(x)\right)\right) .
$$

Consequently to find $u(t, x)$ for given $t>0$ and $x \in \mathbb{R}^{d}$, all we need is to simulate $M$ independent copies $\left(\phi_{0, t}^{-1}(x)\right)^{[j]}$ of $\phi_{0, t}^{-1}(x)$. In fact, when the Law of Large Numbers is 
applied to the sequence $f\left(\left(\phi_{0, t}^{-1}(x)\right)^{[j]}\right)$ the approximations $u^{[M]}(t, x)$ to $u(t, x)$ are obtained by:

$$
u^{[M]}(t, x)=\frac{1}{M} \sum_{j=1}^{M} f\left(\left(\phi_{0, t}^{-1}(x)\right)^{[j]}\right) .
$$

Note that $u^{[M]}(t, x) \rightarrow u(t, x)$ as $N \rightarrow \infty$, almost surely. This is the Monte Carlo approximation to $u(t, x)$ based on the representation (2)-(3). In practice one must approximate the $\left(\phi_{0, t}^{-1}(x)\right)^{[j]}$. In that follows we will show how to do this.

\subsection{Computing approximations to $\phi_{0, t}^{-1}(x)$}

The key stone to approximate $\phi_{0, t}^{-1}(x)$ is find a $\mathrm{RDE}$ having $\phi_{0, t}^{-1}(x)$ as its solution. We follow [2] to deduce this RDE. Let $\phi_{s, t}^{-1}(y)$ the value such that beginning with the initial condition $X(s)=\phi_{s, t}^{-1}(y)$ the solution of

$$
\begin{aligned}
d X(t) & =b(t, X(t)) d t+d W(t) \\
X(0) & =x .
\end{aligned}
$$

in time $t$ satisfies $X(t)=y$. Then, by using the properties of stochastic flows [9] we get

$$
y=\phi_{s, t}^{-1}(y)+\int_{s}^{t} b\left(r, \phi_{r, t}^{-1}(y)\right) d r+\int_{s}^{t} d W(r) .
$$

Hence $\phi_{s, t}^{-1}(y)$ satisfies the -backward in time- stochastic differential equation (SDE)

$$
\phi_{s, t}^{-1}(y)=y-\int_{s}^{t} b\left(r, \phi_{r, t}^{-1}(y)\right) d r-\int_{s}^{t} d W(r), \quad s \in[0, t]
$$

Let us now transform the final-value problem above into a forward-time problem.

For this, define $R(s)=y(t-s), 0 \leq s \leq t$, then

$$
\begin{aligned}
R(s) & =x-\int_{t-s}^{t} b(r, R(t-r)) d r-\int_{t-s}^{t} d W(r) \\
& =x-\int_{0}^{s} b(t-u, R(u)) d u-\xi(s ; \omega) .
\end{aligned}
$$

with $\xi(s ; \omega)=W(t)-W(t-s)$, (here $\omega \in \Omega$ is the realization corresponding to the Brownian path) 
Because the process $\xi(s ; \omega)$ is the difference between the Wiener process in two points, $R(s)$ is at most continuous but not differentiable in $s$. Hence, in order to handle this equation, by defining

$$
Z(s)=R(s)+\xi(s ; \omega)
$$

it is obtained that $Z(s)$ satisfies

$$
Z(s)=x+\int_{0}^{s} g(u, Z(u), \xi(u ; \omega)) d u
$$

where

$$
g(u, Z(u), \xi(u ; \omega)):=-b(t-u, Z(u)-\xi(u ; \omega)) .
$$

Since the mapping $u \rightarrow g(u, Z(u), \xi(u ; \omega))$ is continuous, the right-hand side of the integral equation above is pathwise differentiable. Thus, $Z(s)$ is an stochastic processes on the interval $[0, t]$ with continuously differentiable sample paths. In fact, $Z(s)$ is solution of the Random Differential Equation (RDE) (see, [2])

$$
\begin{aligned}
Z^{\prime}(s) & =g(s, Z(s), \xi(s ; \omega)) \\
Z(0) & =x
\end{aligned}
$$

This is essentially an non-autonomous ODE for almost all realization $\omega \in \Omega$. For the sake of simplicity, from now on we will work with $\xi(s)$ i.e., without an explicit reference to the realization $\omega$.

Note that since we are interested in $\phi_{0, t}^{-1}(x)$. From the equalities

$$
\phi_{0, t}^{-1}(x)=y(0)=R(t)=Z(t)-\xi(t)=Z(t)-W(t),
$$

we conclude that to compute $v(t, x)$ by means of (3) all we need is to obtain the solution of $(5)$ in $s=t$.

In general, it is necessary to resort to numerical method to solve RDEs. The numerical analysis of this kind of equations has not been as widely developed as for deterministic differential equations and even as for SDEs. Nevertheless, in the last years some methods have appeared in the literature for the numerical treatment of RDEs. We refer to [6] for a recent review of the main integrators available.

\section{The proposed integrator}

Since to approximate $u(t, x)=\mathbb{E}(f(Z(t)-W(t)))$ will be required several copies of the solution process to the RDE (5), we are interested in explicit and easy to implement numerical schemes. In that follows we first consider a numerical method for (5) and then we will propose the integrator for (1). 


\subsection{A random Euler method}

For Ordinary Differential Equations with only measurable vector field, the Carathéodory Euler scheme is known to be a convergent method (see, [7] and Section 1.4 of [6]). We can adapt this method to the RDE (5), resulting in a convergent method, as follows.

Let $(\tau)_{h}: t_{0}<t_{1}<\ldots<t_{N}=t$ be a partition of the time interval $[0, t]$ with, for simplicity, equidistant stepsize $h<1$, i.e., defined as a sequence of times such that $t_{n}=t_{0}+n h$, for $n=0,1, \ldots, N$. Starting from the initial value $x$, the approximations $\left\{Z_{i}\right\}$ to $\left\{Z\left(t_{i}\right)\right\},(i=1,2, \ldots, N)$ are obtained recursively by

$$
Z_{n+1}=Z_{n}-h b\left(t-t_{n}-h R_{n}, Z_{n}-\xi\left(t_{n}+h R_{n}\right)\right)
$$

where $R_{n} \sim$ Uniform $\left[\begin{array}{ll}0 & 1\end{array}\right]$.

\subsection{The definitive algorithm}

Finally, the numerical integrator for computing the approximation to $u(t, x)$ in (1) can be algorithmically described as follows:

1. Set the step-size $h=\frac{t}{N}$ (with $N \in \mathbb{N}$ ), set $Z_{0}=x$, and set $M \in \mathbb{N}$ (for the Monte Carlo simulations)

2. Repeat from $j=1$ until $j=M$ :

(a) From $i=0$ until $i=N-1$,

i. generate the Gaussian variable $\eta_{i} \sim N(0,1)$

ii. generate the Uniform random variable $R_{i} \sim \operatorname{Uniform}\left[\begin{array}{ll}0 & 1\end{array}\right]$

iii. compute

$$
\left.Z_{i+1}=Z_{i}-h b\left(t-t_{i}-h R_{i}, Z_{i}-\sqrt{t_{i}+h R_{i}} \eta_{i}\right)\right)
$$

(b) Compute $v^{[j]}=f\left(Z_{N}-\sqrt{t} \eta_{N}\right)$ with $\eta_{N} \sim N(0,1)$

3. Then, $u^{[M]}(t, x)=\frac{1}{M} \sum_{j=1}^{M} v^{[j]}$ is the numerical approximation to $u(t, x)$.

\subsection{Convergence}

Concerning the convergence of the proposed method, we have the following result.

Theorem: Let $u(t, x)$ be the solution to the convection-diffusion PDE (1), with $b$ measurable, locally Lipschitz in the second argument and $\alpha$-Hölder continuous in space uniformly in time, for some $\alpha \in(0,1)$. Let $h<1$ and $M \in \mathbb{N}$ with $M \geqslant \frac{1}{h}$. Then, $u^{[M]}(t, x)$ is almost surely convergent to $u(t, x)$ and we have that

$$
\left|u(t, x)-u^{[M]}(t, x)\right|=O\left(h^{\frac{1}{2}}\right), \quad \text { almost surely }
$$




\section{Conclusions}

In this work we propose an explicit probabilistic numerical integrator for the integration of the deterministic convection-diffusion PDE (1), with non-smooth coefficient $b$. The strategy consisted in representing the solution of the PDE through the expectation of the solution of an associated stochastic transport equation which in turn was solved by a random Euler scheme. In contrast with other kind of stochastic representation for (1), our proposal allows to integrate a more wide class of convection-diffusion PDEs without considering the standard assumptions that are needed for instance in representations based on the Feynman-Kac formulas. We point out that the this approach could be adapted to devising layer methods for some nonlinear convection-diffusion PDEs.

\section{References}

[1] L. Arnold. Stochastic Differential Equations: Theory and Applications. John Wiley \& Sons. 1974.

[2] H. de la Cruz and C. Olivera. A numerical scheme for the integration of the stochastic transport equation. Submitted 2019.

[3] D. Duffy. Finite Difference Methods in Financial Engineering: A Partial Differential Equation Approach. John Wiley \& Sons Ltd. 2006.

[4] R. Durret. Brownian Motion and Martingales in Analysis. Wadsworth Mathematical Series. 1984.

[5] F. Flandoli, M. Gubinelli and E. Priola. Well-posedness of the transport equation by stochastic perturbation. Invent. Math. 180 (1) 1-53, 2010.

[6] X. Han and P. Kloeden. Random Ordinary Differential Equations and Their Numerical Solution. Springer. 2017.

[7] A. Jentzen and A. Neuenkirch. A random Euler scheme for Carathéodory differential equation. JCAM 224. Issue 1. 346-359. 2009.

[8] B. S. Jovanović and E. Süli. Analysis of Finite Difference Schemes for Linear Partial Differential Equations with Generalized Solutions. Springer-Verlag. 2014.

[9] H. Kunita. Stochastic flows and stochastic differential equations. Cambridge University Press. 1990.

[10] K. W. Morton. Numerical Solution of Convection-Diffusion Problems. Chapman and Hall. London. 1996. 